\title{
Screening for subtelomeric chromosome abnormalities in children with idiopathic mental retardation using multiprobe telomeric FISH and the new MAPH telomeric assay
}

\author{
Carolina Sismani ${ }^{1}$, John AL Armour ${ }^{2}$, Jonathan Flint ${ }^{3}$, Christina Girgalli ${ }^{1}$, Regina Regan ${ }^{3}$ \\ and Philippos C Patsalis*,1 \\ ${ }^{1}$ Department of Cytogenetics, The Cyprus Institute of Neurology and Genetics, P.O. BOX 23462, 1683 Nicosia, \\ Cyprus; ${ }^{2}$ Institute of Genetics, University of Nottingham, Queen's Medical Centre, Nottingham, NG7 2UH UK; \\ ${ }^{3}$ Institute of Molecular Medicine, John Radcliffe Hospital, Headington, Oxford, UK
}

\begin{abstract}
Subtelomeric chromosomal abnormalities are emerging as an important cause of human genetic disorders. The scope of this investigation was to screen a selected group of children with idiopathic mental retardation for subtelomeric anomalies using the multiprobe telomeric FISH method and also to develop and test a new assay, the MAPH telomeric assay, in the same group of patients. The new MAPH telomeric assay uses the recently published MAPH methodology that permits the measurement of locus copy number by hybridisation with a specifically designed set of probes located at the end of human chromosomes. Seventy patients with idiopathic mental retardation have been screened using the established multiprobe telomeric FISH assay and the new MAPH telomeric assay, for all telomeres. One patient with de novo 8p subtelomeric deletion was identified. The new MAPH telomeric assay confirmed the same results in both normal and abnormal samples. This is the first description of the use of MAPH methodology to detect chromosomal imbalances near the telomeres in idiopathic mentally retarded patients. The new MAPH telomeric assay offers a new, fast, accurate and cost effective diagnostic tool to detect chromosomal imbalances near telomeres in mentally retarded patients, as well as the characterisation of known chromosomal abnormalities, spontaneous recurrent miscarriages, infertility, hematological malignancies, preimplantation genetic diagnosis, and other fields of clinical and research interests. European Journal of Human Genetics (2001) 9, 527-532.
\end{abstract}

Keywords: Subtelomeric; mental retardation; FISH; MAPH

\section{Introduction}

Mental retardation is a common and distressing disorder that affects $3 \%$ of the population and its origins are poorly understood. ${ }^{1}$ Our poor understanding of its origins hampers the provision of effective treatment and preventive regimens, and remains a major challenge for medical practice. The

*Correspondence: PC Patsalis, Department of Cytogenetics, The Cyprus Institute of Neurology and Genetics, P.O.BOX 23462, 1683 Nicosia, Cyprus. Tel: +357 2 392695; Fax: +357 2 392793;

E-mail: patsalis@mdrtc.cing.ac.cy

Received 21 January 2001; revised 20 April 2001; accepted 26 April 2001 cause of moderate and severe mental retardation is chromosomal or monogenenic in $30-40 \%$ of cases, environmental factors explain a further $10-30 \%$ and for about $40 \%$ the cause is still unknown. ${ }^{2,3}$ In the case of mild mental retardation, chromosomal anomalies and environmental causes explain about $30 \%$ of cases and for the remaining $70 \%$ the cause is unknown. ${ }^{4,5}$ Standard cytogenetic analysis in children with mental retardation is usually performed by G-banding at an ISCN 550 G-band level. This method is capable of detecting DNA rearrangements of 5-10 Mb or larger which is a limiting factor for the detection of subtle rearrangements in patients. Hypervariable DNA polymorph- 
isms (HVPs), detected by probes for variable number of tandem repeats (VNTRs) can be used to find cryptic chromosomal anomalies by observation of abnormal inheritance of alleles from the parents. ${ }^{6}$ By using this method, several investigations revealed submicroscopic chromosomal deletions and rearrangements in subjects who have an apparently normal karyotype and thus provide evidence that such anomalies are an important unrecognised cause of mental retardation. ${ }^{7,8}$

As an alternative to whole genome screening for submicroscopic anomalies, two methods for detecting telomeric rearrangements have been developed and applied to investigate such anomalies in patients with idiopathic mental retardation. The first method relies on detecting deviation from Mendelian inheritance of alleles at polymorphic loci close to telomeres. ${ }^{6,8}$ Two studies have reported frequencies of 6 and $7.4 \%$ subtelomeric rearrangements in individuals reported as normal after routine cytogenetic analysis. ${ }^{8,9}$ The second method exploits FISH using telomeric probes on metaphase chromosomes, to assess directly whether there is a loss or gain of telomere copy number. ${ }^{10,11}$ The largest study to date, by Knight et al using the multiprobe telomere FISH approach in 284 children with unexplained moderate to severe mental retardation and 182 children with mild retardation, indicated that subtle chromosome rearrangements occurred in 7.4 and $0.5 \%$ of cases respectively. ${ }^{12}$ Telomere screening is a very useful diagnostic test and the first step towards the goal of high resolution analysis of the entire genome for chromosome rearrangements. ${ }^{13}$

This study presents a new methodology for detecting subtelomeric abnormalities, namely the MAPH telomeric assay, which exploits the recently published MAPH with a new specifically designed set of telomeric probes. MAPH (Multiplex Amplifiable Probe Hybridisation) methodology permits the accurate determination of locus copy number in the genome. ${ }^{14}$ Briefly, in this method a set of specifically designed probes, all flanked by the same primer-binding sites, is hybridised with the test DNA. After washing, specifically bound probes are detected by amplification using the primers flanking the probe region. Since an excess of probe is used, the amount of probe amplified should reflect the copy number of the corresponding locus in the sample. ${ }^{14}$

This is the first description of the use of MAPH telomeric assay, as a fast, accurate and cost effective methodology to detect chromosomal imbalances near telomeres in mentally retarded patients, as compared with multiprobe telomeric FISH.

\section{Materials and methods \\ Sample selection}

A group of 70 unrelated individuals aged 4-20 with idiopathic mental retardation were included in this study. All of them fulfill the following three criteria: (a) Normal karyotype: Cytogenetic analysis was done at a $>550$ band level using standard procedures; (b) Normal for fragile $\mathrm{X}$ syndrome: PCR and/or Southern blot analysis for all samples were done in the CING Cytogenetic Department; ${ }^{15}$ (c) Without having a clinically recognisable syndrome: All patients were examined and referred by the genetics clinic of Makarios III Medical Center as idiopathic mental retardation without having a clinically recognisable syndrome. Dysmorphic features, family history and other clinical information were also referred. Among the 70 patients, 42 have mild mental retardation and 28 have moderate or severe mental retardation. In addition, for the development of the MAPH assay, DNA samples were used from (a) 25 unrelated unaffected controls, (b) 20 samples with known subtelomeric chromosomal rearrangements and (c) four samples with known numerical chromosomal abnormalities.

\section{Multiprobe telomeric FISH assay}

Fixed chromosome suspensions for FISH analysis were prepared from peripheral blood using standard procedures. The probe labelling, preparation of slide devices and the multiprobe FISH protocol were performed as previously described by Knight et al. ${ }^{10}$ A complete set of all of the telomere specific clones was kindly provided to us by Dr J Flint (Table 1). FISH analyses were done using the Cytovision ChromoFluor $^{\mathrm{TM}}$ analyser (Applied Image Co.) and all images were saved on optical disks.

\section{MAPH telomeric assay}

The new MAPH telomeric assay uses the recently published MAPH methodology and a specifically designed set of probes located at the end of 41 human chromosomes (Table 1). The MAPH probes were prepared using insert DNAs from the same telomere specific clones that were used in multiprobe FISH (Table 1), except the probes for XpYp, which were prepared by PCR from published sequence. ${ }^{16}$ Insert fragments from PAC clones were initially separated from the vectors using $30 \mathrm{U}$ of Not $\mathrm{I}$ and $30 \mathrm{U}$ of MluI, then run on $1 \%$ low melting agarose and recovered using $\beta$-Agarase. They were then digested with $A l u \mathrm{I}$, dephosphorylated with $20 \mathrm{U}$ of Calf Intestinal Alkaline Phosphatase and finally run on a $1.5 \%$ agarose gel, against a 20 bp ladder for size-selection. Agarose blocks containing fragments from 100-800 bp were cut out and purified with Qiagen kit (Qiagen Inc). The telomeric fragments were subcloned into the EcoRV site of pZero-2 (Invitrogen) and transformed into E. coli TOP 10 by electroporation. Single colonies from the transformed plates were transferred to microtitre plates. Replica nylon filters were hybridised with $20 \mathrm{ng}{ }^{32} \mathrm{P}$-labelled genomic DNA to identify clones containing multicopy sequences. ${ }^{17}$ Selected single copy probes were amplified directly from the bacterial cultures using the common primers PZA, and PZB. ${ }^{14}$ Products were initially run on a $3 \%$ agarose gel for the initial sizing and those in the range of $140-700 \mathrm{bp}$ were selected, end-labelled with ${ }^{33} \mathrm{P}$ and run on a $6 \%$ denaturing polyacrylamide gel for 
Table 1 Probes for multiprobe FISH and MAPH telomeric assays

\begin{tabular}{|c|c|c|c|c|c|c|c|}
\hline & Multip & FISH telomer & bes & & MAPH & neric probes & \\
\hline Telomere & Clone name & Clone type & Reference & Clone name & Size $b p$ & Reliability & Reference \\
\hline $1 p$ & CEB108 & Cosmid & 24 & ST3A3 & 297 & Informative & This report \\
\hline & & & & ST3A2 & 273 & Informative & This report \\
\hline $2 p$ & $2052 f 6$ & Cosmid & 11 & ST13A5 & 380 & Informative & This report \\
\hline $2 q$ & $210 \mathrm{E} 14$ & Cosmid & 11 & ST4A4 & 237 & Informative & This report \\
\hline $3 p$ & B47a2 & Cosmid & 11 & ST4C3 & 393 & Informative & This report \\
\hline $4 p$ & B31 & Cosmid & 19 & ST14A6 & 178 & Unreliable & This report \\
\hline & & & & ST14A2 & 480 & Informative & This report \\
\hline $4 q$ & CT55 & Cosmid & 20 & ST2F5 & 259 & Informative & This report \\
\hline $5 p$ & $114 J 18$ & BAC & 21 & ST12A6 & 370 & Unreliable & This report \\
\hline $5 q$ & B22a4 & Cosmid & 11 & ST13B3 & 232 & Unreliable & This report \\
\hline $6 p$ & $62 \mid 11$ & PAC & 21 & ST1A1 & 283 & Informative & This report \\
\hline $6 q$ & 571124 & PAC & 21 & ST1H8 & 162 & Informative & This report \\
\hline $9 q$ & $224 a 1$ & Cosmid & 10 & ST10C3 & 495 & Informative & This report \\
\hline $10 p$ & $2189 \mathrm{~b} 6$ & Cosmid & 11 & $\mathrm{ST} 14 \mathrm{H} 4$ & 141 & Informative & This report \\
\hline $10 q$ & 2136a1 & Cosmid & 10 & ST13C4 & 245 & Informative & This report \\
\hline $11 p$ & $2209 a 2$ & Cosmid & 11 & ST6C4 & 269 & Informative & This report \\
\hline $11 q$ & 2072cl & Cosmid & 11 & ST11C2 & 310 & Informative & This report \\
\hline $12 p$ & $496 a 11$ & PAC & 21 & ST13C6 & 280 & Informative & This report \\
\hline $12 q$ & $2196 \mathrm{~b} 2$ & Cosmid & 11 & ST7B2 & 509 & Informative & This report \\
\hline $13 q$ & 85A10 & PAC & 11 & ST11D5 & 221 & Informative & This report \\
\hline $14 q$ & 2006a1 & Cosmid & 11 & ST7D3 & 308 & Unreliable & This report \\
\hline $15 q$ & 154P1 & PAC & 11 & ST5G1 & 137 & Informative & This report \\
\hline $16 p$ & CGG4 & Cosmid & 11 & ST6E3 & 253 & Informative & This report \\
\hline $16 q$ & D3b1 & Cosmid & 11 & ST10C7 & 307 & Informative & This report \\
\hline $17 p$ & 211b1 & Cosmid & 10 & ST10C12 & 374 & Informative & This report \\
\hline $17 q$ & B37cl & Cosmid & 11 & ST10D1 & 255 & Informative & This report \\
\hline & & & & $\mathrm{ST} 11 \mathrm{H} 8$ & 220 & Informative & This report \\
\hline $21 q$ & C9a1 & Cosmid & 11 & ST $15 C 9$ & 263 & Unreliable & This report \\
\hline & & & & ST $15 B 6$ & 358 & Unreliable & This report \\
\hline & & & & ST15C10 & 147 & Informative & This report \\
\hline $22 q$ & N85A3 & Cosmid & 22 & ST9A3 & 407 & Informative & This report \\
\hline & & & & ST9A6 & 336 & Informative & This report \\
\hline XpYp & CY29 & Cosmid & 23 & ST 16A1 & 144 & Polymorphic & This report \\
\hline & & & & ST 16A2 & 156 & Polymorphic & This report \\
\hline & & & & ST $16 \mathrm{~A} 4$ & 192 & Polymorphic & This report \\
\hline & & & & ST $16 \mathrm{~A} 6$ & 133 & Polymorphic & This report \\
\hline & & & & ST $16 \mathrm{~A} 8$ & 445 & Polymorphic & This report \\
\hline $\mathrm{XgYg}$ & C8.1/1 & Cosmid & 23 & ST $15 G 2$ & 220 & Unreliable & This report \\
\hline
\end{tabular}

more accurate sizing. A minimum of one probe per chromosome end was selected for each and two mixes were developed with differently sized probes, namely ST1 and ST2 with 27 and 28 probes respectively (Table 1). MAPH methodology was carried out as originally described. ${ }^{14}$

\section{Results}

Multiprobe telomeric FISH

All of the 70 tested samples had at least $90 \%$ good quality metaphases within the hybridised area and good hybridisations were obtained. A minimum of five metaphases with 
interpretable hybridisation signals was analysed for every chromosome end. Among the 70 idiopathic mentally retarded children tested, one case was identified with a subtelomeric deletion on the p-arm of chromosome 8 (Figure 1 ). The patient is 5 years old and she has moderate mental retardation, microcephaly, obesity, speech delay and hypotonia of central origin. Retrospectively, the subtle 8p deletion was detected by high-resolution cytogenetic analysis. The deletion appeared de novo and was not present in either parent. In all of the above cases and in all other cases with inconclusive results, FISH analysis was repeated at least twice.

\section{MAPH telomeric assay}

We have developed specifically designed sets of 55 probes (ST1/ST2) required for the MAPH telomeric assay. The 55 probes, flanked by the same common primers PZA, and $\mathrm{PZB},{ }^{14}$ represent unique subtelomeric loci. In order to test the specificity, reliability and informativeness of these probes, hybridisations were performed using the set ST1/ ST2 with target DNA from four groups of samples: (a) 25 normal controls; (b) 20 samples with known subtelomeric chromosomal rearrangements; (c) four samples with known numerical chromosomal abnormalities; and (d) 60 out of the 70 samples with idiopathic mental retardation that were previously tested with multiprobe telomeric FISH and DNA was available. Following hybridisation, the probes from the

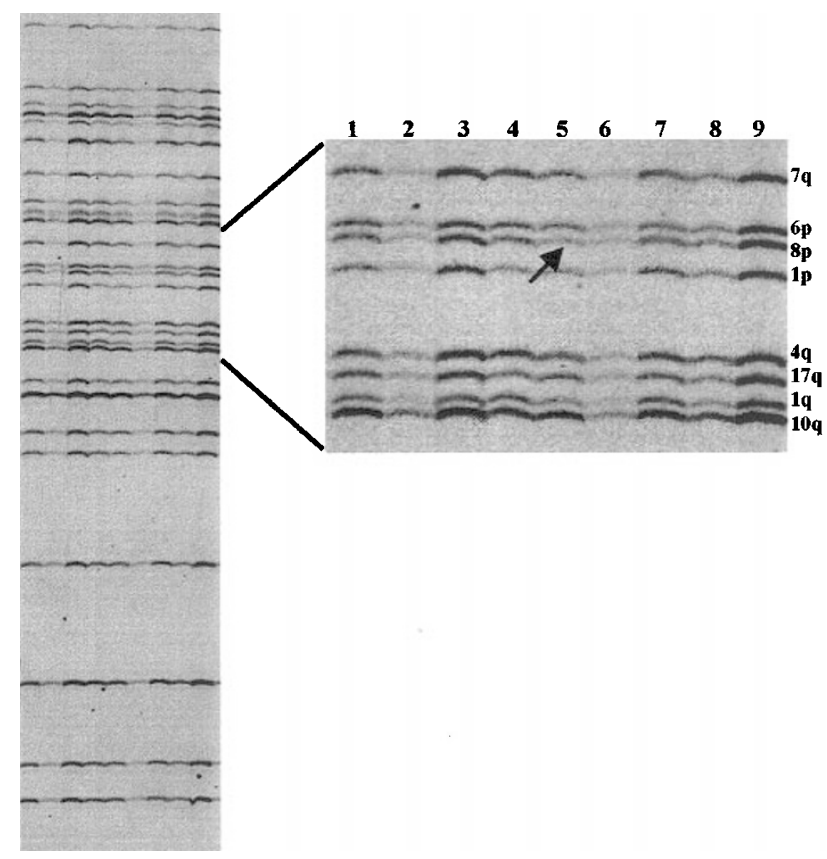

Figure 1 Gel analysis from a MAPH experiment with ST2 mix, showing the amplification of recovered probes from nine idiopathic mentally retarded individuals. Individual five is the IMR female with the heterozygous subtelomeric deletion of $8 p$. This deletion was identified by the reduced signal intensity of the corresponding probe. Arrow shows the deleted locus.
ST1/ST2 set were recovered and amplified quantitatively. Relative band intensities for every probe in all samples were compared by calculating the normalised ratios as described in Armour et al. ${ }^{14}$ A threshold ratio was set at 0.75 and thus all reliable and specific probes were expected to give ratio values between 0.75 and 1.25 . After quantitative analysis of the data obtained from the 25 normal samples (group a), 51 probes appeared to be reliable and specific with constant relative band intensities as shown by the normalised ratios $(\mathrm{nr}=1.0 \pm 0.25)$ obtained in all samples. Further characterization of the probes by sequence analysis as well as using samples bearing known cytogenetic abnormalities showed 10 probes to be unreliable (Table 1), either because sequence analysis showed them to be from the wrong subtelomeric region $(5 p, 20 p)$, or because unacceptably variable signals were obtained in the absence of true copy number change (1q, 14 A6 from 4p, 5q, 9p, 14q, $15 \mathrm{C} 9$ and 15B6 from 21q, and XqYq). Furthermore, copy number polymorphism for the XpYp was detected, with at least two variant alleles encountered. The commoner variant allele at XpYp ( $\mathrm{f} \approx$ amp;0.06) deleted 16A4 and 16A8, the rarer deleted all the XpYp probes shown in Table 1. A blind test using 20 samples with previously characterised cytogenetic abnormalities (group b) correctly identified 15 out of 16 deletions and seven out of 13 duplications. This analysis correctly identified anomalies on chromosomes $1 \mathrm{p}, 1 \mathrm{q}, 4 \mathrm{p}, 6 \mathrm{q}, 7 \mathrm{q}, 8 \mathrm{p}$, $9 q, 11 p, 12 p, 13 q, 16 q, 18 q, 19 p, 19 q, 22 q$ and failed to identified on chromosomes 2q, 9p, 6q, 10q, 20p, XYq. All abnormalities were correctly identified in four samples with numerical abnormalities (group c), consisting of $[47, X X+21],[47, X Y+13],[47, X X Y]$ and $[45, X]$. Finally, DNA samples from 60 out of 70 patients with idiopathic mental retardation that were previously examined with multiprobe telomere FISH (group d), were finally investigated in a blind study aimed to further characterise the probes and confirm the results obtained by multiprobe telomere FISH. The subtelomeric $8 \mathrm{p}$ deletion was easily detected by MAPH telomeric assay as the signal intensity of probe $15 \mathrm{C} 3$ from the $8 \mathrm{p}$ clone, was reduced (n.r=0.65) (Figure 2). Normalised ratios obtained from this probe from all other 59 samples were constant and clustered around 1.0. The results from the blind study obtained from MAPH telomeric assay confirmed both the samples with $8 \mathrm{p}$ deletion and all the other samples as normal. In summary, by combining our results from the four different experiments, we have a MAPH set with reliable, specific, single copy and informative probes from 33 ends.

\section{Discussion}

We report the results of a multiprobe FISH telomeric screening in a well-defined group of 70 patients aged $4-20$ with idiopathic mental retardation, as well as the development and testing of the new MAPH telomeric assay in the same group of patients. 


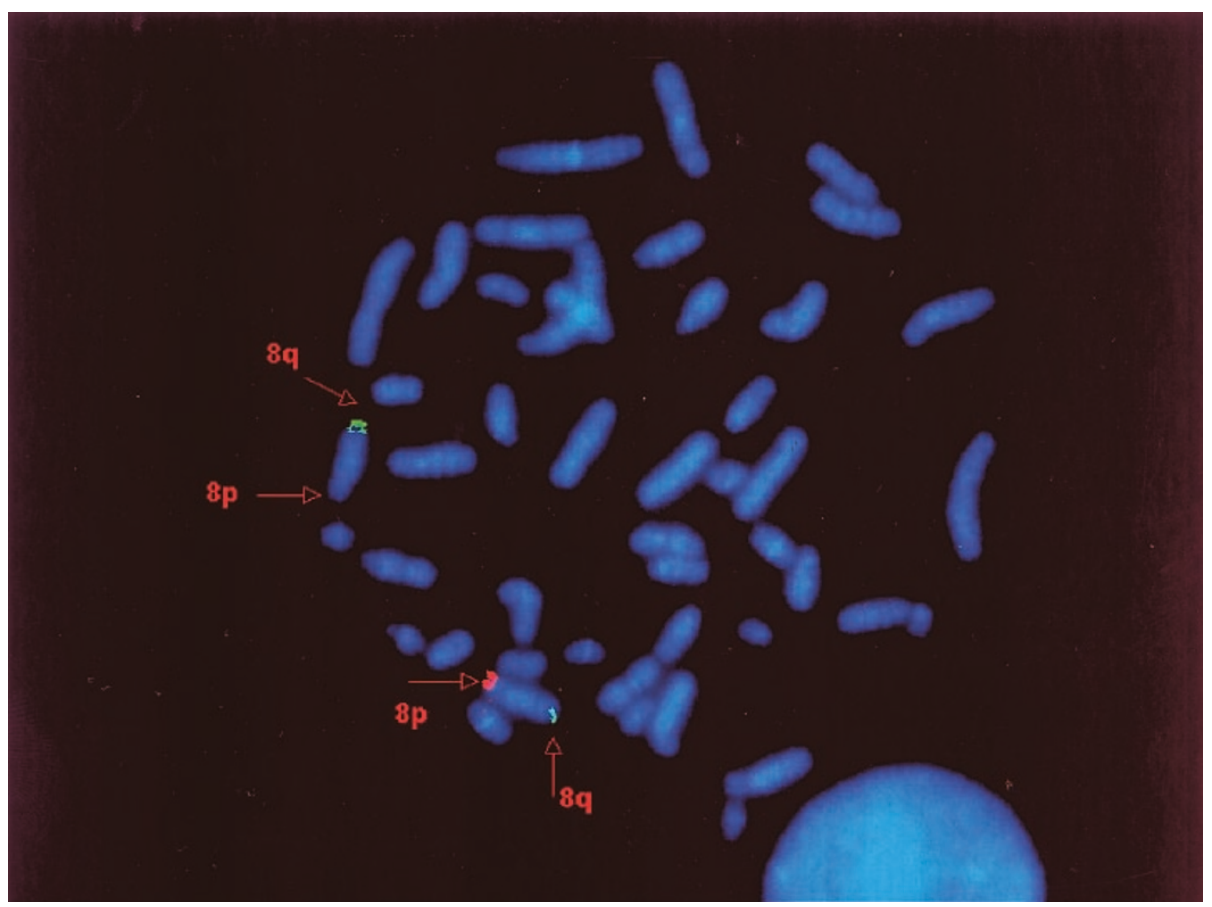

Figure 2 Multiprobe FISH results, showing a hybridised metaphase from the idiopathic mental retardation female detected to have a de novo deletion of the subtelomeric region of chromosome $8 \mathrm{p}$. P-arm signals appearing red and q-arms green.

This investigation is important as it provides information for the efficiency of screening of a clinically well-defined group of patients. Furthermore, the results are important for advancing our understanding of the pathogenesis of mental retardation and for the clinical management of affected children. This screening identified one child with moderate mental retardation, with a de novo 8pter subtelomeric deletion using both multiprobe telomeric FISH and MAPH telomeric assays independently. In the present study, the observed prevalence of subtelomeric chromosomal abnormalities is $3.6 \%$ (one out of 28 : $95 \%$ confidence intervals $0-$ $10.4 \%$ ) among moderate to severe idiopathic mental retardation patients and $0 \%$ (0 out of 42 : $95 \%$ confidence intervals $0-7.1 \%$ ) among mild idiopathic mental retardation patients. The study of 70 subjects is not sufficient to estimate the frequency precisely, but is within the confidence limits observed in other studies. ${ }^{12,13}$

Multiprobe telomeric FISH screening assay is currently the method of choice for the detection of subtelomeric abnormalities. $^{9-11,13,18}$ However, it is a very time-consuming, expensive and difficult technique that makes it hard to be adopted in routine screening or performed as a service by genetic diagnostic laboratories. We report, for the first time, the development of a new fast and cost effective method, the MAPH telomeric assay. This is the first test of the new assay and further development, testing and experience is clearly needed to optimise the efficiency and accuracy of the method. The first set of specifically designed probes ST1/
ST2 for all telomeres proved partially insufficient with only 33 of the 41 ends being screened with confidence. In spite of the incomplete informativeness of the ST1/ST2 probe set used in this pilot study, the telomeric MAPH assay confirmed both the normal and the one abnormal result among the patients tested. Despite the relatively low informativeness of the ST1/ST2 probe set used in this pilot study, screening with MAPH telomeric assay would be a cost-effective way to making diagnoses in children with unexplained mental retardation. Work in progress is aiming to assemble a set of MAPH probes (ST3) which can screen all 41 unique subtelomeric regions in a single assay, and this set will be further tested on a large number of well-defined groups of idiopathic mentally retarded patients.

In summary, we have screened a group of 70 children with unexplained mental retardation and determined one subtelomeric anomaly using the well established multiprobe telomeric FISH method and an innovative MAPH telomeric assay independently. The development and testing of the new MAPH telomeric assay offer a new fast and cost effective diagnostic tool for the investigation of mental retardation, the characterisation of known chromosomal abnormalities, spontaneous recurrent miscarriages, infertility, hematological malignancies, preimplantation genetic diagnosis, the investigation of human telomere structure and function, identification of dosage sensitive genes involved in human genetic diseases and other fields of clinical and research interests. 


\section{Acknowledgments}

We would like to acknowledge The Wellcome Trust and the Cyprus Institute of Neurology and Genetics for financial support, Dr V Anastasiadou and Dr G Stylianidou for their assistance.

\section{References}

1 Birch HG, Richardson SA, Baird D, Horobin G, Ilsley R: Mental subnormality in the community: a clinical and epidemiological study. Baltimore: Williams and Wilkins, 1970.

2 Gustavson KH, Hagberg B, Hagberg G, Sars K: Severe mental retardation in a Swedish country: II, etiologic and pathogenetic aspects of children born 1959-1970. Neuropadiatrie 1977; 8: $293-304$.

3 Laxova R, Ridler MAC, Bowen-Bravery M: An etiological survey of the severely retarded Hertfordshire children who were born between January 1, 1965 and December 31, 1967. Am J Med Genet 1977; 1: $75-86$.

4 Lamont MA, Dennis NR: Aetiology of mild mental retardation. Arch Dis Child 1988; 63: 1032-1038.

5 Einfeld SL: Clinical assessment of 4500 developmentally delayed individuals. J Med Defic Res 1984; 28: 129-142.

6 Wilkie AOM: Detection of cryptic chromosomal abnormalities in unexplained mental retardation: a general strategy using hypervariable subtelomeric DNA polymorphisms. Am J Hum Genet 1993; 53: $688-701$

7 Lamb J, Wilkie AOM, Harris PC et al: Detection of breakpoints in submicroscopic chromosomal translocation, illustrating an important mechanism for genetic disease. Lancet 1989; ii: $819-824$.

8 Flint J, Wilkie AO, Buckle VJ, Winter RM, Holland AJ, McDermid HE: The detection of subtelomeric chromosomal rearrangements in idiopathic mental retardation. Nat Genet 1995; 9: 132 140.

9 Slavotinek A, Rosenberg M, Knight S et al: Screening for submicroscopic chromosome rearrangements in children with idiopathic mental retardation using microsatellite markers for the chromosome telomeres. J Med Genet 1999; 36: 405 - 411.

10 Knight SJL, Horsley SW, Regan R et al: Development and clinical application of an innovative fluorescence in situ hybridization technique which detects submicroscopic rearrangements involving telomeres. Eur J Hum Genet 1997; 5: 1-8.
11 National Institute of Health and Institute of Molecular Medicine. Collaboration. A complete set of telomeric probes and their clinical application. Nat Genet 1996; 14: 86-89.

12 Knight SJL, Regan R, Nicod A et al: Subtle chromosomal rearrangements in children with unexplained mental retardation. The Lancet 1999; 354: 1676-1681.

13 Knight SJL, Flint J: Perfect endings: a review of subtelomeric probes and their use in clinical diagnosis. J Med Genet 2000; 37: $401-409$.

14 Armour JAL, Sismani C, Patsalis PC, Cross G: Measurement of locus copy number by hybridization with amplifiable probes. Nucleic Acids Res 2000; 28: 2: 605 - 609.

15 Patsalis PC, Sismani C, Hettinger J et al: Molecular screening of fragile X (FRAXA) and FRAXE mental retardation syndromes in the Hellenic population of Greece and Cyprus: Incidence, genetic variation and stability. Am J Med Genet 1999; 84: 184-190.

16 Baird DM, Royle NJ: Sequences from higher primates orthologous to the human $\mathrm{Xp} / \mathrm{Yp}$ telomere junction region reveal gross rearrangements and high levels of divergence. Hum Mol Genet 1997; 13: 2291-2299.

17 Maniatis T, Sambrook J, Fritsch EF: Molecular Cloning. Cold Spring Harbor Laboratory Press, 1989.

18 Borgione E, Giudice ML, Gelesi O et al: How microsatellite analysis can be exploited for subtelomeric chromosomal rearrangements analysis in mental retardation. I Med Genet 2001; 38: E1.

19 Youngman S, Bates GP, Williams S et al: The telomeric regions on 13p, 15p, 21p, and 22p. Genomics 1992; 14: 350-356.

20 Wright TJ, Wijmenga C, Clark LN, Frants RR, Williamson R, Hewitt JE: Fine Mapping of the FSHD gene region orientates the rearranged fragment detected by the probe p13E-11. Hum Mol Genet 1993; 2: 1673-1678.

21 Knight SJL, Lese CM, Precht KS et al: An optimized set of human telomere clones for studing telomere integrity and architecture. Am J Hum Genet 2000; 67: 320-332.

22 Nesslinger NJ, Gorski JL, Kurczynski TW et al: Clinical characterization of seven patients with deletions of chromosome 22q13.3. Am J Hum Genet 1994; 54: 464-472.

23 Kvaloy K, Galvagni F, Brown WR: The sequence organization of the long arm pseudoautosomal region of the human sex chromosomes. Hum Mol Genet 1994; 3: 771-778.

24 Giraudeau F, Aubert D, Young I et al: Molecular cytogenetic detection of 1p36.3. J Med Genet 1997; 34: 314-317. 